\title{
Long-term results of the surgical management of insulinoma patients with MEN1: a Groupe d'étude des Tumeurs Endocrines (GTE) retrospective study
}

\author{
Delphine Vezzosi, Catherine Cardot-Bauters ${ }^{1}$, Nicolas Bouscaren $^{2}$, Maëlle Lebras $^{3}$, \\ Mireille Bertholon-Grégoire ${ }^{4}$, Patricia Niccoli ${ }^{5}$, Nathalie Levy-Bohbot ${ }^{6}$, \\ Lionel Groussin? ${ }^{7}$ Philippe Bouchard ${ }^{8}$, Antoine Tabarin ${ }^{9}$, Philippe Chanson ${ }^{10}$, \\ Pierre Lecomte ${ }^{11}$, Isabelle Guilhem ${ }^{12}$, Nicolas Carrere ${ }^{13}$, Eric Mirallié ${ }^{14}$, \\ François Pattou ${ }^{15}$, Jean Louis Peix ${ }^{16}$, Diane Goere ${ }^{17}$, Françoise Borson-Chazot ${ }^{4}$, \\ Philippe Caron, Vanina Bongard ${ }^{2}$, Bruno Carnaille ${ }^{15}$, Pierre Goudet ${ }^{18}$ \\ and Eric Baudin ${ }^{19}$ \\ Service d'Endocrinologie, Maladies Métaboliques et Nutrition, Centre Hospitalier Universitaire Rangueil-Larrey, \\ Université Paul Sabatier et INSERM U1037, Toulouse, France, ${ }^{1} \mathrm{CHRU}$ de Lille, Clinique Marc-Linquette, Service \\ d'Endocrinologie-Métabolisme, Lille, France, ${ }^{2}$ Service d'Epidémiologie, Centre Hospitalier Universitaire, Toulouse, \\ France, ${ }^{3}$ Clinique d'Endocrinologie, Centre Hospitalier Universitaire, Nantes, France, ${ }^{4}$ Service d'Endocrinologie, \\ Centre Hospitalier Est, Hospices Civils de Lyon, Université Lyon 1 et INSERM U1052, Lyon, France, \\ ${ }^{5}$ Service d'Endocrinologie, Diabète et Maladies Métaboliques, Centre Hospitalier Universitaire La Timone, \\ Marseille, France, 'Service d'Endocrinologie, Centre Hospitalier Universitaire de Reims, Hôpital Robert Debré, \\ Reims, France, ${ }^{7}$ Service d'Endocrinologie, Centre Hospitalier Universitaire de Cochin, Paris, France, \\ ${ }^{8}$ Service d'Endocrinologie, Groupement Hospitalier Universitaire Est, Hôpital Saint Antoine, Paris, France, \\ ${ }^{9}$ Service d'Endocrinologie, Centre Hospitalier Universitaire, Hôpital du Haut Levêque, Pessac, France, \\ ${ }^{10}$ Service d'Endocrinologie et des Maladies de la Reproduction, Hôpitaux Universitaires Paris-Sud, Hôpital de \\ Bicêtre, Le Kremlin-Bicêtre, France, ${ }^{11}$ Unité END, CHRU Bretonneau, Tours, France, ${ }^{12}$ Service d'Endocrinologie, \\ Diabète et Maladies Métaboliques, CHU de Rennes, Hôpital Sud, Rennes, France, ${ }^{13}$ Service de Chirurgie digestive, \\ CHU Purpan, Toulouse, France, ${ }^{14} \mathrm{Clinique}$ de Chirurgie Digestive et Endocrinienne, CHU Nantes, Nantes, France, \\ ${ }^{15}$ Service de Chirurgie Générale et Endocrinienne, CHU Lille, Lille Cedex, France, ${ }^{16}$ Service de Chirurgie \\ Endocrinienne, Hospices Civils de Lyon, Centre Hospitalier Lyon Sud, Pierre Bénite, France, \\ ${ }^{17}$ Service de Chirurgie Oncologique, Institut Gustave Roussy, Villejuif, France, ${ }^{18}$ Service de Chirurgie \\ Endocrinienne, Centre Hospitalier Universitaire de Dijon, Dijon, France and ${ }^{19}$ Service de Médecine Nucléaire \\ et de Cancérologie Endocrine, Institut Gustave Roussy, Villejuif, France
}

Correspondence
should be addressed
to E Baudin
Email
eric.baudin@gustaveroussy.fr

\begin{abstract}
Objective: Management of insulinomas in the context of MEN1 remains poorly studied. The aim of this study was to evaluate long-term results of various surgical approaches in a large cohort of insulinoma-MEN1 patients.

Design and methods: Consecutive insulinoma-MEN1 patients operated on for a nonmetastatic insulinoma between 1957 and 2010 were retrospectively selected from the MEN1 database of the French Endocrine Tumor Group. The type of surgery was categorized as distal pancreatectomy (DP), total pancreatectomy/cephalic duodenopancreatectomy (TP/CDP), or enucleation (E). Primary endpoint was time until recurrence of hypoglycemia after initial surgery. Secondary endpoints were post-operative complications.

Results: The study included 73 patients (median age $=28$ years). Surgical procedures were DP $(n=46), \operatorname{TP} / \operatorname{CDP}(n=9)$, or $\mathrm{E}(n=18)$. After a median post-operative follow-up of 9.0 years (inter-quartile range (IQR): 2.5-16.5 years), 60/73 patients (82.2\%) remained hypoglycemia free. E and TP/CDP were associated with a higher risk of recurrent hypoglycemia episodes (unadjusted hazard ratio: 6.18 ((95\% Cl: 1.54-24.8); $P=0.010)$ for E vs DP and 9.51 ((95\% Cl: $1.85-48.8) ; P=0.007)$ for TP/CDP vs DP. After adjustment for International Union against Cancer pTNM classification, enucleation remained significantly associated with a higher probability of recurrence. Long-term complications had occurred in $20(43.5 \%)$ patients
\end{abstract}


with DP, five (55.6\%) with TP/CDP, but in none of the patients who have undergone $E(P=0.002)$.

Conclusion: In the French Endocrine database, DP is associated with a lower risk for recurrent hypoglycemia episodes. Due to lower morbidity, E alone might be considered as an alternative.

\section{Introduction}

About 5\% of pancreatic neuroendocrine neoplasms (pNEN) arise as part of a MEN1 syndrome $(1,2,3,4,5)$. Insulinomas are the second most common functioning tumor after gastrinomas and account for about 10-30\% of pNEN-MEN1 $(6,7,8,9)$. Surgical removal is recommended because of the risk of severe hypoglycemia including neurological damage during the long-term management and an unknown effect of medical management in the long run $(6,9,10,11,12,13)$. However, no specific surgical procedure is recommended by European Neuroendocrine Tumor Society (ENETS) guidelines in insulinomas-MEN1 patients (14). By consequence, controversies exist regarding the extent of surgery which is performed in these patients. Distal pancreatectomy (DP) is considered to be the procedure of choice as it yields a high rate of cure in several series $(5,10,11,15,16,17,18,19,20,21)$. However, DP exposes patients to the risk of secondary diabetes and exocrine secretion deficit especially in the context of MEN1, for which additional surgery is frequently required $(22,23)$. Enucleation (E) may be an attractive pancreatic parenchyma-sparing alternative strategy and is proposed by several authors in the case of a single or dominant pNEN $(24,25)$. Indeed, long-term cure has been reported using this procedure (25). However, the lack of large series of patients with a long-term followup makes it difficult to evaluate the benefit-risk ratio of both strategies. Until now, only one series of insulinomas in the context of MEN1 has been published with more than 20 patients and a long-term follow-up exceeding 10 years (6). This signifies that surgical strategies continue to be poorly evaluated in insulinoma patients with MEN1.

The purpose of our multicentric, retrospective study of the French Groupe des Tumeurs Endocrines was twofold: i) to determine risk of recurrence of hypoglycemia after initial surgery in a large series of well-characterized insulinoma-MEN1 patients according to the type of surgery and ii) to search for short- and long-term complications.

\section{Patients and methods}

\section{Patients}

The Endocrine Tumor Group network for MEN1, created in February 1991, comprises clinical centers spread over the 22 regions of France and four genetics departments. Seven hundred and forty-one MEN1 patients were registered in the French MEN1 database between 1957 and 2010. Out of these 741 patients, all consecutive patients with insulinoma and MEN1 included in the French MEN1 database were sought. The medical records were retrospectively reviewed by one investigator on site (D V).

Inclusion criteria were the following: MEN1 diagnostic criteria as defined by the guidelines (at least two of the three major MEN1-correlated disorders or patients with one typical disorder and a positive family history or patients with the identification of a germline mutation in the MEN1 gene) (26) and a diagnosis of insulinoma based on the presence of symptoms of hypoglycemia associated with low plasma glucose concentrations $(\leq 0.45 \mathrm{~g} / \mathrm{l})$ and inappropriately high serum insulin $(\geq 3 \mathrm{mUI} / \mathrm{l})$ or C-peptide $(\geq 0.6 \mathrm{ng} / \mathrm{ml}$ ) level (27). Exclusion criteria were the presence of synchronous distant metastases at diagnosis and patients unamenable to surgery.

The following items had been recorded at the diagnosis of insulinoma: age, gender, World Health Organization (WHO) 2004 classification (28), International Union against Cancer (UICC) (29) and ENETS pTNM classifications (30), the presence of other functioning or nonfunctioning pancreatic neoplasms, number and diameter of pNENs, hormone staining characteristics, and date of first surgery. The WHO 2010 classification could not be analyzed due to insufficient reporting on the proliferative index over the study period. The patients followed up clinically had undergone appropriate blood tests at least once a year. During follow-up, the following criteria had been recorded until December 31, 2010 or the date of the last follow-up: hypoglycemia-symptom 
status, date of recurrent hypoglycemic symptoms, and presence of other functioning or nonfunctioning pancreatic neoplasms. Genetic studies had been performed after informed consent had been provided by each patient in accordance with French law.

\section{Imaging studies}

Preoperative imaging studies after 1980 included a pancreatic computed tomography (CT) scan and/or a pancreatic magnetic resonance imaging in all patients and, an endoscopic ultrasound in 29 patients. In the 16 patients diagnosed with insulinoma before 1980, CT had been performed in five patients, visceral arteriography in two patients, and no preoperative radiological examination had been performed in nine patients. Portal venous sampling for insulin or calcium angiogram was performed in eight patients, localizing region(s) secreting insulin in four patients. Intraoperative ultrasound was performed in 16 out of 73 patients.

The preoperative location of the primary NENs had been recorded. Postoperative imaging with at least one abdominal CT scan had been performed in all patients every $1-3$ years.

\section{Therapeutic management}

For all patients, surgery was performed within the neuroendocrine tumor French national network. The surgical procedure had been recorded and categorized in three groups: total pancreatectomy (TP)/cephalic duodeno-pancreatectomy (CDP), DP extending to the portal vein combined or not with other NEN enucleations, or tumor enucleation alone (E). TP and CDP were gathered in the same group because of the low number of patients in each group. In addition, the results of the surgical procedures were analyzed in two subgroups: DP alone or E of the body or tail of the pancreas.

Bidigital palpation of the pancreas was performed in 67 out of 73 patients. Duodenotomy was performed in only one patient with concomitant gastrin secretion.

After surgery, in addition to the pTNM status, the residual tumor classification had been recorded and defined as R0 in case of no residual tumor, R1 in case of microscopic residual tumor, and $\mathrm{R} 2$ in case of macroscopic residual tumor. The size, location, and number of NEN with insulin-positive staining had been recorded, as well as the presence of other NENs, at the pathological analysis.

Postoperative adverse effects had been recorded and classified according to the Common Terminology Criteria for Adverse Events, version 4.0 (grade $1=$ mild symptoms with no intervention required; grade $2=$ moderate symptoms with minimal, local or noninvasive intervention indicated; grade $3=$ severe or medically significant symptoms but not immediately life-threatening; grade $4=$ life threatening consequences; and grade $5=$ death). Short-term complications were defined as those occurring before 6 months postoperatively, otherwise they were classified as long-term complications. The risk of longterm complications was analyzed by decade categorized as: before 1980, 1980-1990, 1991-2000, and 2001-2010.

\section{Endpoint and statistics}

The main endpoint of this study was time until recurrence of hypoglycemia defined as the time between the first surgical procedure and the recurrence of clinical symptoms together with concomitant hypoglycemia. The presence of insulin-positive staining NENs at pathology was not used to define a curative surgery, but only the presence or absence of hypoglycemic symptoms and results of plasma glucose, serum insulin, and C-peptide levels at the time of hypoglycemic symptoms in all the 42 patients who were operated after 1990. Patients who experienced persistent hypoglycemia within 3 months after the surgery were classified as 'not cured' by surgery, otherwise they were classified as cured regardless of the recurrence status after surgery.

Data analysis was conducted on SAS Statistical Software (SAS Institute, Cary, NC, USA, release 9.2). The characteristics of the patients selected for each type of surgical intervention were compared for all parameters recorded. All quantitative data were expressed as medians (inter-quartile range (IQR)). The groups were compared at baseline using the $\chi^{2}$ or Fisher's exact test for qualitative data and the Kruskall-Wallis test for quantitative data. Hypoglycemia-free survival curves for each surgical category were plotted using the Kaplan-Meier method and compared with the Log rank test. The description of the length of follow-up and the delay until recurrence of hypoglycemia resulted from the Kaplan-Meier estimation. Cox regression was used to model the risk of recurrent hypoglycemia episodes after treatment according to baseline characteristics and surgical treatment and to estimate hazard ratios together with $95 \%$ CIs. The patients were censored at the time of recurrent hypoglycemia episodes or at the end of the last follow-up if they remained free of symptoms at that time. The duration of follow-up was considered null for patients 'not cured' after surgery. The proportional-hazard assumption was tested for each 


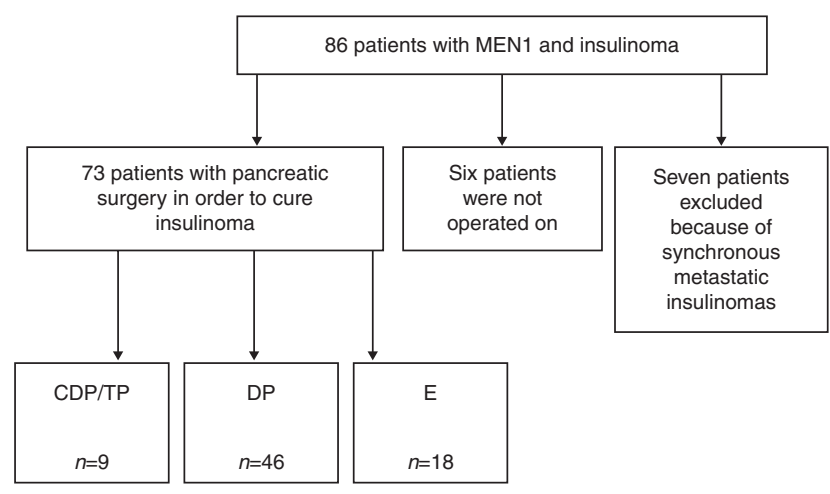

Figure 1

Study flowchart. CDP, cephalic duodenopancreatectomy; TP, total pancreatectomy; DP, distal pancreatectomy; E, enucleation alone.

independent variable by the 'log-log' method plotting $(-\ln (-\ln ($ survival $)))$ for each category of a nominal variable vs $\ln$ (analysis time). The assumption could not be rejected for any variable. Because of the small number of events, adjustment had to be limited to UICC pTNM classification. The variables associated with long-term complications were assessed with $\chi^{2}$, Fisher's, or KruskallWallis test. A $P$ value $<0.05$ was considered to be statistically significant.

\section{Results}

\section{Population characteristics}

The medical records of 86 patients with insulinoma and MEN1, followed up in 30 French centers, were analyzed (Fig. 1). Among these 86 patients, seven (8.1\%) had presented with distant metastases at diagnosis and were excluded. In addition, the records of six patients who had never been operated on were excluded. Finally, 73 patients operated on for a nonmetastatic insulinoma within the context of MEN1 constitute the study subjects. The period of time when pancreatic surgery had been performed was distributed as follows: before 1980 for 16 patients, 1980-1990 for 15 patients, 1991-2000 for 25 patients, and 2001-2010 for 17 patients.

The main initial characteristics of the 73 operated patients with nonmetastatic insulinoma are summarized in Table 1. Briefly, there were 47 (64.4\%) women and $26(35.6 \%)$ men whose median age at surgery was 28 years (IQR: 17-39). The median follow-up time was 9.0 years (IQR: $2.5-16.5)$. At the time of surgery, 14 patients (19.2\%) had another functioning pNEN (gastrinomas, $n=12$;
GHRHomas with acromegalic symptoms, $n=2$ ). An extra-pancreatic tumor of the MEN1 spectrum had already been diagnosed in 48 patients $(65.7 \%)$ including hyperparathyroidism $(n=28)$, pituitary adenoma $(n=14)$, adrenal adenomas $(n=7)$, and others extra-pancreatic lesions $(n=6)$ (total exceeding 48 because some patients had several extra-pancreatic tumors). The familial history was found in 51 cases $(69.9 \%)$ and MEN1 mutation in 61 cases $(83.6 \%)$.

At the end of the follow-up, seven patients were deceased: one non-neuroendocrine lung cancer, three thymic carcinoids, one ependymoma, one pituitary sarcoma, and one pulmonary embolism 8 months after a TP. No MEN1 insulinoma-related death was reported.

Table 1 Characteristics of the 73 MEN1-insulinoma patients at the time of surgery.

\begin{tabular}{l}
\hline Parameters \\
\hline Male gender, $n$ (\%) \\
Median age at diagnosis (years) \\
Date of first surgery \\
$<1980$ \\
$1980-1990$ \\
$1991-2000$ \\
$2001-2010$ \\
Extra-pancreatic MEN1 tumor \\
Other functioning pancreatic NET \\
Preoperative location of pancreatic NET \\
Median insulin-positive NETs - largest \\
diameter (mm) \\
Multiple insulin-positive \\
NETs at pathology, $n$ (\%) \\
Location of the largest insulin-positive NETs \\
Tail \\
Body \\
Head \\
Number of NET at pathology \\
1 \\
2 \\
$\geq 3$ \\
2004 WHO classification \\
Benign \\
Uncertain behavior \\
UICC pTNM classification \\
T1-T2 \\
ENETS pTNM classification \\
T1-T2 \\
Median duration of follow-up after \\
surgery (years) \\
\end{tabular}

$\frac{\text { Total } n=73}{26(35.6 \%)}$

$16(21.9 \%)$

$15(20.5 \%)$

$25(34.2 \%)$

$17(23.3 \%)$

$48(65.7 \%)$

$14(19.2 \%)$

$62(84.9 \%)$

20 (IQR: 15-25)

$23(37.1 \%)$

$38(52.0 \%)$

$19(26.0 \%)$

$16(21.9 \%)$

$26(35.6 \%)$

$13(17.8 \%)$

$34(46.6 \%)$

$32(43.8 \%)$

$41(56.2 \%)$

$67(91.8 \%)$

$6(8.2 \%)$

$73(100 \%)$

9.0 (IQR: 2.5-16.5)

CDP/TP, cephalic duodenopancreatectomy/total pancreatectomy; IQR inter-quartile range; $D P$, distal pancreatectomy; $E$, enucleation alone; ENETS, European Neuroendocrine Tumor Society; UICC, International Union against Cancer; WHO classification, World Health Organization classification.

${ }^{\text {a }}$ Missing for 15 patients.

${ }^{\mathrm{b}}$ Missing for 11 patients. 
Table 2 Surgical procedures performed in the 73 insulinoma-MEN1 patients.

\begin{tabular}{|c|c|c|c|c|}
\hline \multirow[b]{2}{*}{ Parameters } & \multicolumn{4}{|c|}{ Type of surgery } \\
\hline & $\mathrm{E} n=18$ & $\mathrm{CDP} / \mathrm{TP} n=9$ & DP $n=46$ & $P$ value \\
\hline Male gender, $n(\%)$ & $6(33.3 \%)$ & $4(44.4 \%)$ & $16(34.8 \%)$ & 0.835 \\
\hline Median age at diagnosis (years) & 22 (IQR: 15-34) & 35 (IQR: 33-48) & 29 (IQR: 17-39) & 0.045 \\
\hline \multicolumn{5}{|l|}{ Date of first surgery } \\
\hline$<1980$ & $5(27.8 \%)$ & $1(11.1 \%)$ & $10(21.7 \%)$ & 0.803 \\
\hline $1980-1990$ & $3(16.7 \%)$ & $2(22.2 \%)$ & $10(21.7 \%)$ & \\
\hline $1991-2000$ & $6(33.3 \%)$ & $2(22.2 \%)$ & $17(37.0 \%)$ & \\
\hline $2001-2010$ & $4(22.2 \%)$ & $4(44.4 \%)$ & $9(19.6 \%)$ & \\
\hline Extra-pancreatic MEN1 tumor & $11(61.1 \%)$ & $6(66.7 \%)$ & $31(67.4 \%)$ & 0.891 \\
\hline Other functioning pancreatic NEN & $2(11.1 \%)$ & $3(33.3 \%)$ & $9(19.6 \%)$ & 0.414 \\
\hline Preoperative location of NEN & $13(72.2 \%)$ & $8(88.9 \%)$ & $41(89.1 \%)$ & 0.229 \\
\hline $\begin{array}{l}\text { Median insulin positive NEN - largest } \\
\text { diameter }(\mathrm{mm})^{\mathrm{a}}\end{array}$ & 20 (IQR: 10-25) & 27.5 (IQR: 17.5-55) & 20 (IQR: 15-23) & 0.205 \\
\hline $\begin{array}{l}\text { Multiple insulin positive NEN at } \\
\text { histopathology, } n(\%)^{\mathrm{b}}\end{array}$ & $4(23.5 \%)$ & $3(37.5 \%)$ & $16(43.2 \%)$ & 0.379 \\
\hline \multicolumn{5}{|c|}{ Location of the largest insulin positive NEN } \\
\hline Tail & $10(55.6 \%)$ & $2(22.2 \%)$ & $26(56.5 \%)$ & 0.042 \\
\hline Body & $5(27.8 \%)$ & $1(11.1 \%)$ & $13(28.3 \%)$ & \\
\hline Head & $3(16.7 \%)$ & $6(66.7 \%)$ & $7(15.2 \%)$ & \\
\hline \multicolumn{5}{|l|}{ Number of pancreatic NEN at pathology } \\
\hline 1 & $11(61.1 \%)$ & $4(44.4 \%)$ & $11(23.9 \%)$ & 0.003 \\
\hline 2 & $5(27.8 \%)$ & $1(11.1 \%)$ & $7(15.2 \%)$ & \\
\hline \multirow{2}{*}{\multicolumn{5}{|c|}{2004 WHO classification }} \\
\hline & & & & \\
\hline Benign & $11(61.1 \%)$ & $3(33.3 \%)$ & $18(39.1 \%)$ & 0.223 \\
\hline Uncertain behavior & $7(38.9 \%)$ & $6(66.7 \%)$ & $28(60.9 \%)$ & \\
\hline \multicolumn{5}{|l|}{ UICC pTNM classification } \\
\hline $\mathrm{T} 1-\mathrm{T} 2$ & $17(94.4 \%)$ & $7(77.8 \%)$ & $43(93.5 \%)$ & 0.285 \\
\hline $\mathrm{T} 3$ & $1(5.6 \%)$ & $2(22.2 \%)$ & $3(6.5 \%)$ & \\
\hline \multicolumn{5}{|l|}{ ENETS pTNM classification } \\
\hline $\mathrm{T} 1-\mathrm{T} 2$ & $18(100 \%)$ & $9(100 \%)$ & $46(100 \%)$ & NA \\
\hline $\mathrm{T} 3$ & 0 & 0 & 0 & \\
\hline $\begin{array}{l}\text { Median duration of follow-up after } \\
\text { surgery (years) }\end{array}$ & 6.5 (IQR: 1.0-12.2) & 3.0 (IQR: 0.3-9.0) & 10.0 (IQR: 4.7-19.2) & 0.124 \\
\hline
\end{tabular}

CDP/TP, cephalic duodenopancreatectomy/total pancreatectomy; IQR, inter-quartile range; DP, distal pancreatectomy; E, enucleation alone; ENETS, European Neuroendocrine Tumor Society; UICC, International Union against Cancer; WHO classification, World Health Organization classification; NA, not applicable.

a Missing for 15 patients.

${ }^{\mathrm{b}}$ Missing for 11 patients.

\section{Surgical procedures}

Surgical procedures performed in the 73 patients were the following: enucleation alone (E) $(n=18), \mathrm{DP}(n=46)$, and $\mathrm{TP} /$ cephalic duodenopancreatectomy (TP/CDP) $(n=9)$ (Fig. 1 and Table 2). In the 18 patients who had undergone an enucleation alone, multiple enucleations had been performed in three patients. In the 46 patients who had undergone a DP, enucleation of additional tumors in the pancreatic head had been performed in 12 patients whereas in the six patients who had undergone CDP, enucleation from the pancreatic tail had been performed in one patient. The reasons for TP were the following: more than five pancreatic tumors (five tumors in one patient, 16 tumors in one patient, and eight tumors in one patient) and difficulty to perform enucleation in the head of the pancreas because of the vicinity of the vessels. A laparoscopic approach was performed in five out of 18 patients all treated with enucleation alone (27.8\% of this subgroup).

A lymph node dissection had been performed in 12 patients (16\%) whose tumor size was below or above two centimeters in five and seven patients respectively. The median number of resected nodes was three (IQR: two to ten nodes) according to both the surgical and pathology reports. Due to the low number of lymphadenectomy, the $\mathrm{N}$ status was not considered in the statistical analysis.

According to the type of surgery, patients who had undergone $\mathrm{E}$ were statistically younger $(P<0.05)$, and had a lower number of NEN at pathology $(P=0.003)$ (Table 2$)$. Largest NEN was more frequently located in the head in case of CDP/TP. No other confounding parameter was found. 
No peroperative mortality had occurred. One patient had died 8 months after a TP due to a pulmonary embolism.

\section{Pathological results}

One-hundred and ten NENs with insulin-positive staining were found in 73 patients. The median number of resected NENs with insulin-positive staining was one (IQR: 1-2). Multiple NENs with insulin-positive staining were found in 23 patients (37.1\%) $(n=2$ in 15 patients, $n=3$ in five patients, $n=4$ in one patient, and $n \geq 5$ in two patients) (Table 1). Taking into account all pNEN, the median number of resected tumors was one (IQR: $1-4$ ). The sites of the largest NENs with insulin-positive staining in each patient were distributed as follows: head $(n=16)$, body $(n=19)$, and tail $(n=38)$. The median diameter of the largest NENs with insulin-positive staining was $20 \mathrm{~mm}$ (IQR: $15-25 \mathrm{~mm}$ ) including the largest diameter below $10 \mathrm{~mm}$ and above $30 \mathrm{~mm}$ in 11 and ten patients respectively. All NENs with insulin-positive staining were well differentiated. According to the WHO 2004 classification, NENs with insulin-positive staining were considered to be benign in 32 patients or of uncertain behavior in 41 patients. In these 41 patients with lesions classified as of uncertain behavior, 35 had NENs exceeding $2 \mathrm{~cm}$ and six exhibited angioinvasion. According to the pTNM UICC classification, 35 tumors were T1, 32 T2, and six T3. According to the pTNM ENETS classification, 35 tumors were T1 and 38 were T2. Resection was considered to be R0 in 70 patients and R1 in three patients. The type of surgery was DP in two patients and $\mathrm{E}$ in one patient among the three patients whose resection was defined as R1. No positive lymph node metastasis was found among the 12 patients who had undergone a lymphadenectomy.

\section{Recurrence of hypoglycemia}

After a median follow-up of 9.0 years (IQR: $2.5-16.7$ years), $60 / 73$ patients (82.2\%) had remained hypoglycemia-free after surgery. Thirteen patients had experienced recurrent hypoglycemia episodes including three patients classified as 'not cured' by surgery. The pathological findings of these three patients were as follows: multiple NENs with insulin-positive staining in one patient; benign according to the WHO 2004 classification in three patients; T1Nx or T2Nx according to the pTN UICC or ENETS classifications in two or one patient(s) respectively; R1 resection status in all three. DP and $\mathrm{E}$ were performed in respectively two and one patients.
T3 tumor as defined by UICC pTNM classification was significantly associated with the risk of recurrent hypoglycemia, as shown by unadjusted Cox analysis (Table 3). T1-T2 or T3 tumors were associated with recurrent hypoglycemia episodes in $14.9 \%$ or $50.0 \%$ of cases respectively. The unadjusted hazard ratio for recurrent hypoglycemia episodes was 4.54 (95\% CI: 1.22-16.9, $P=0.024)$ for T3 vs T1-T2 (Table 3).

DP, TP/CDP, or E were significantly associated with the recurrence of hypoglycemia in $8.7 \%$ (4/46), 33.3\% (3/9), and $33.3 \%(6 / 18)$ of cases respectively corresponding to hazard ratios (HR) of 9.51 ((95\% CI: $1.85-48.8)$, $P=0.007)$ for TP/CDP compared with DP or 6.18 ( $(95 \% \mathrm{CI}$ : 1.54-24.8), $P=0.010$ ) for E compared with DP (Table 3). Hypoglycemia symptom-free survival is represented according to surgical treatment shown in Fig. 2. Of note, among the 46 patients who underwent a DP, all 12 patients who had undergone DP and enucleation of additional tumors in the pancreatic head had remained hypoglycemia free. By contrast, four out of $34(11 \%)$ patients who underwent a DP without enucleation of additional tumors in the pancreatic head recurred.

Among the nine patients who underwent TP/CDP, all the three patients who recurred were treated by CDP. Recurrence of hypoglycemia after enucleation did not significantly differ $(P=0.999)$ between patients treated with open surgery (four patients out of 13 , corresponding to $30.8 \%$ of recurrence) and those treated with laparoscopy (two patients out of five, corresponding to $40.0 \%$ of recurrence).

After adjustment for UICC pTNM classification, hazard ratios were $\mathrm{HR}=6.75((1.66-27.5) ; P=0.008)$ for $\mathrm{E}$ vs DP or $\mathrm{HR}=5.27((0.85-32.7) ; P=0.074)$ for $\mathrm{TP} / \mathrm{CDP}$ vs DP (Table 3). The median time to recurrence of hypoglycemia was 10.0 years (IQR: 4.7-19.2 years) for DP compared with 6.5 years (1.0-12.2 years) for $\mathrm{E}$, and 3.0 years (0.3-9.0 years) for TP/CDP, $P=0.002$ (Log rank test).

The same analysis was performed in the subgroup of 34 patients who underwent DP, only, and compared with the 18 patients who underwent $\mathrm{E}$ of the body or tail of the pancreas. DP or E was associated with the recurrence of hypoglycemia in $11.8 \%$ (4/34) or $33.3 \%$ (6/18) of cases, respectively corresponding to hazard ratios of 4.52 ((95\% CI: 1.13-18.1), $P=0.033)$ for E compared with DP.

\section{Short- and long-term surgical complications}

The short-term complications had been recorded in 24 patients (32.9\%) and had occurred after $\mathrm{E}$ in four patients 
Table 3 Short- and long-term recurrent hypoglycemia episodes at the end of follow-up. Hazard ratios for continuous variables are given for a one unit increase.

\begin{tabular}{|c|c|c|c|c|c|}
\hline Parameters & $\begin{array}{c}\text { Recurrence of } \\
\text { hypoglycemia after } \\
\text { surgery } n(\%)\end{array}$ & $\begin{array}{c}\text { Hazard ratio } \\
\text { unadjusted }(95 \% \mathrm{Cl})\end{array}$ & $P$ value & $\begin{array}{c}\text { Hazard ratio } \\
\text { adjusted }^{\mathrm{a}}(95 \% \mathrm{Cl})\end{array}$ & $P$ value \\
\hline \multicolumn{6}{|l|}{ Gender, $n(\%)$} \\
\hline Female & $7 / 47(14.9 \%)$ & 1 & 0.567 & & \\
\hline Male & $6 / 26(23.1 \%)$ & $1.38(0.46-4.11)$ & & & \\
\hline Age at diagnosis (years) & $N A^{b}$ & $0.97(0.93-1.02)$ & 0.245 & & \\
\hline \multicolumn{6}{|l|}{ Date of first surgery } \\
\hline$\leq 1990$ & $9 / 31(29 \%)$ & 1 & 0.699 & & \\
\hline $1991-2010$ & $4 / 42(9.5 \%)$ & $0.78(0.22-2.80)$ & & & \\
\hline \multicolumn{6}{|l|}{ Extra-pancreatic MEN1 tumor } \\
\hline No & $8 / 25(32.0 \%)$ & 1 & & & \\
\hline Yes & $5 / 48(10.4 \%)$ & $0.63(0.19-2.05)$ & 0.438 & & \\
\hline \multicolumn{6}{|l|}{ Other functioning pancreatic NEN } \\
\hline No & $9 / 59(15.2 \%)$ & 1 & & & \\
\hline Yes & $4 / 14(28.6 \%)$ & $1.79(0.54-5.95)$ & 0.343 & & \\
\hline \multicolumn{6}{|l|}{ Preoperative location of pancreatic NENs } \\
\hline No & $3 / 11(27.3 \%)$ & 1 & & & \\
\hline Yes & $10 / 62(16.1 \%)$ & $0.81(0.22-2.98)$ & 0.753 & & \\
\hline Insulin-positive NEN - largest diameter $(\mathrm{mm})^{\mathrm{c}}$ & $N A^{b}$ & $1.02(0.99-1.05)$ & 0.150 & & \\
\hline \multicolumn{6}{|l|}{ Multiple insulin-positive NEN at pathology, $n(\%)^{d}$} \\
\hline No & $10 / 39(25.6 \%)$ & 1 & & & \\
\hline Yes & $2 / 23(8.7 \%)$ & $0.31(0.07-1.44)$ & 0.137 & & \\
\hline \multicolumn{6}{|l|}{ Location of the largest insulin-positive NEN } \\
\hline Tail & $6 / 38(15.8 \%)$ & 1 & & & \\
\hline Body & $4 / 19(21.0 \%)$ & $0.95(0.27-3.41)$ & 0.939 & & \\
\hline Head & $3 / 16(18.7 \%)$ & $1.21(0.30-4.91)$ & 0.788 & & \\
\hline \multicolumn{6}{|l|}{ Number of pancreatic NEN at pathology } \\
\hline 1 & $6 / 26(23.1 \%)$ & 1 & & & \\
\hline 2 & $4 / 13(30.8 \%)$ & $1.64(0.45-6.06)$ & 0.455 & & \\
\hline$\geq 3$ & $3 / 34(8.8 \%)$ & $0.52(0.13-2.12)$ & 0.362 & & \\
\hline \multicolumn{6}{|l|}{2004 WHO classification } \\
\hline Benign & $7 / 32(21.9 \%)$ & 1 & & & \\
\hline Uncertain behavior & $6 / 41(14.6 \%)$ & $0.88(0.29-2.68)$ & 0.827 & & \\
\hline \multicolumn{6}{|l|}{ UICC pTNM classification } \\
\hline $\mathrm{T} 1-\mathrm{T} 2$ & $10 / 67(14.9 \%)$ & 1 & & & \\
\hline $\mathrm{T} 3$ & $3 / 6(50.0 \%)$ & $4.54(1.22-16.9)$ & 0.024 & & \\
\hline \multicolumn{6}{|l|}{ ENETS pTNM classification } \\
\hline $\mathrm{T} 1-\mathrm{T} 2$ & $13 / 73(17.8 \%)$ & NA & NA & & \\
\hline $\mathrm{T} 3$ & 0 & & & & \\
\hline \multicolumn{6}{|l|}{ Surgical procedures } \\
\hline $\mathrm{DP}(n=46)$ & $4 / 46(8.7 \%)$ & 1 & & 1 & \\
\hline $\mathrm{CDP} / \mathrm{TP}(n=9)$ & $3 / 9(33.3 \%)$ & $9.51(1.85-48.8)$ & 0.007 & $5.27(0.85-32.7)$ & 0.074 \\
\hline $\mathrm{E}(n=18)$ & $6 / 18(33.3 \%)$ & $6.18(1.54-24.8)$ & 0.010 & $6.75(1.66-27.5)$ & 0.008 \\
\hline
\end{tabular}

(22.2\% of E procedures) (grades $1-2$ : $n=3$, grades $3-4$ : $n=1)$, after CDP/TP in six patients $(66.7 \%$ of $\mathrm{CDP} / \mathrm{TP}$ procedures) (grades 1-2: $n=1$ and grades 3-4: $n=5$ ), and after DP in 14 patients (30.4\% of DP procedures) (grades 1-2: $n=9$, grades 3-4: $n=5)$. These short-term complications included: endocrine insufficiency $(n=10 ; 41.7 \%)$, exocrine insufficiency $(n=5 ; 20.8 \%)$, pancreatic fistulas
( $n=6 ; 25 \%)$, infectious complications $(n=2 ; 8.3 \%)$, acute pancreatitis $(n=2 ; 8.3 \%)$, renal failure $(n=1 ; 4.2 \%)$, and respiratory failure $(n=1 ; 4.2 \%)$.

Long-term complications had been recorded in 25 patients (34.2\%) and had never occurred after E, but five patients had experienced such complications after CDP/TP (55.6\%) (grades 3-4: $n=4$, grade 5: $n=1$ ) 


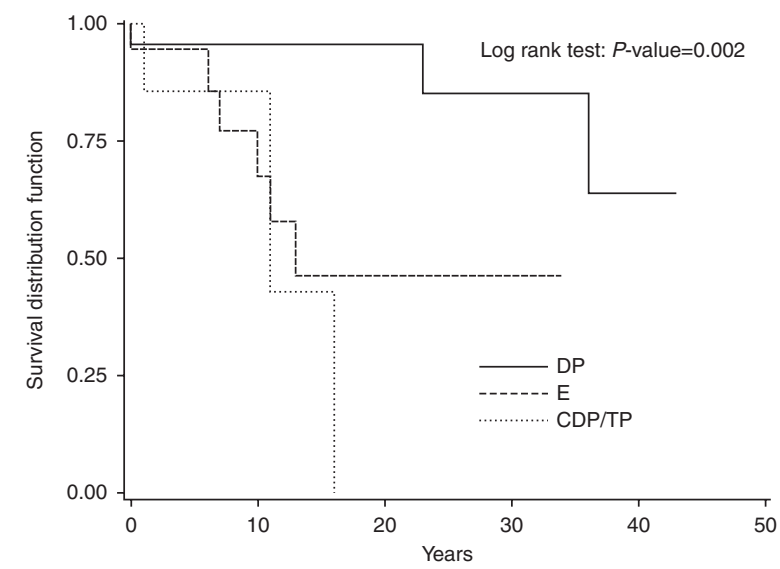

Number at risk

$\begin{array}{lccccc}\text { DP } & 46 & 25 & 11 & 5 & 2 \\ \text { CDP/TP } & 9 & 2 & 0 & 0 & 0 \\ \text { E } & 18 & 7 & 3 & 2 & 0\end{array}$

\section{Figure 2}

Hypoglycemia symptom-free survival in 73 patients operated on for a nonmetastatic pancreatic MEN1-insulinoma as a function of the type of surgery. CDP, cephalic duodenopancreatectomy; TP, total pancreatectomy; DP, distal pancreatectomy; $E$, enucleation alone.

and 20 patients after DP (43.5\%) (grades 1-2: $n=2$, grades 3-4: $n=18$ ) (Table 4). Among the patients with long-term complications, endocrine insufficiency $(n=22$; $88 \%$ of long-term complications), exocrine insufficiency $(n=9 ; 36 \%)$, volvulus of the small intestine $(n=1 ; 4 \%)$, and death as a result of a pulmonary embolism $(n=1)$ were found. Twenty patients had started hypoglycemic agents including insulin therapy in ten cases and oral hypoglycemic agents in ten cases after surgery. Regarding exocrine insufficiency, nine patients had started pancreatic enzyme replacement during the course of the study.

Long-term complications were significantly associated with the presence of multiple positive insulin NENs at pathological analysis $(60.9 \%$ in the group with multiple vs $25.6 \%$ in patients with a solitary positive insulin NEN) $(P=0.006)$, the presence of multiple pNEN at pathology $(15.4 \%$ in patients with only one pNEN vs 30.8 and $50.0 \%$ in those with two and three pNEN respectively) $(P=0.019)$, and the type of surgical procedures $(0,55.6 \%$, and $43.5 \%$ after $\mathrm{E}, \mathrm{CDP} / \mathrm{TP}$, and $\mathrm{DP}$ respectively) $(P=0.002)$. Long-term complications were not significantly affected by the period of time $(P=0.111): 50 \%$ (8/16) occurred before 1980, 40\% (6/15) between 1980 and 1990, 16\% (4/25) between 1991 and 2000, and 41.2\% (7/17) between 2001 and 2010.

\section{Discussion}

To the best of our knowledge, this study is by far the largest series dedicated to the surgical management of insulinoma-MEN1 patients within a national network. Both the high number of patients and the duration of follow-up make it possible to identify the surgical approach allowing the lowest risk for recurrent hypoglycemia episodes in insulinoma-MEN1 patients. InsulinomaMEN1 patients represent $11.6 \%$ of the MEN1 population analyzed in this study. Median age was 28 years, $8.1 \%$ had presented features of a malignant insulinomas, and finally another pNET was present in 19\% of the cases. These figures are in accordance with those reported in previous publications $(10,16,21,31)$.

Some authors proposed a DP associated with enucleation of any additional tumors in the pancreatic head as the treatment of choice to control hypoglycemic symptoms $(6,20,32)$. Even if long-term complications of this procedure in MEN1 patients remain poorly studied, preliminary evidence suggest a high risk of endocrine and/or exocrine insufficiency (33). Progress in the field of parenchyma-preserving resections in pNEN now offer alternative approaches especially in case of single pNEN $(24,25)$. The number of patients included in the previous

Table 4 Long-term surgical complications. The number of complications is greater than the number of patients because some patients experienced multiple complications.

\begin{tabular}{|c|c|c|c|}
\hline & $\begin{array}{l}\text { Number of } \\
\text { patients with } \\
\text { long-term } \\
\text { complications } \\
(\%)\end{array}$ & $\begin{array}{l}\text { Type of long-term } \\
\text { complications } \\
\text { (number of patients) }\end{array}$ & $\begin{array}{c}\text { Grade } \\
\text { (number of } \\
\text { patients) }\end{array}$ \\
\hline $\mathrm{E} n=18$ & 0 & NA & NA \\
\hline \multirow[t]{4}{*}{$\begin{array}{c}\mathrm{CDP} / \mathrm{TP} \\
n=9\end{array}$} & $5(55.6 \%)$ & $\begin{array}{l}\text { Endocrine } \\
\text { insufficiency } \\
\quad(n=4)\end{array}$ & $3(n=4)$ \\
\hline & & $\begin{array}{l}\text { Exocrine } \\
\text { insufficiency } \\
(n=5)\end{array}$ & $3(n=5)$ \\
\hline & & $\begin{array}{l}\text { Volvulus of small } \\
\text { intestine }(n=1)\end{array}$ & $4(n=1)$ \\
\hline & & $\begin{array}{l}\text { Pulmonary } \\
\text { embolism }(n=1)\end{array}$ & $5(n=1)$ \\
\hline \multirow[t]{2}{*}{$\mathrm{DP} n=46$} & $20(43.5 \%)$ & $\begin{array}{l}\text { Endocrine } \\
\quad \text { insufficiency } \\
\quad(n=18)\end{array}$ & $\begin{array}{l}2(n=2) \\
3(n=16)\end{array}$ \\
\hline & & $\begin{array}{l}\text { Exocrine } \\
\quad \text { insufficiency } \\
(n=4)\end{array}$ & $3(n=4)$ \\
\hline
\end{tabular}

E, enucleation; CDP/TP, cephalic duodenopancreatectomy/total pancreatectomy; DP, distal pancreatectomy; NA, not applicable. 
studies was too low to allow a reliable analysis of the best surgical strategy. Especially, only five to eight patients benefit from $\mathrm{E}$ alone, so far.

Our study of 73 patients followed-up over a median of 9.0 years suggests that a DP was associated with the highest symptom-free survival and manageable toxicity. DP was associated with recurrent hypoglycemic episodes in $8.7 \%$ of cases compared with $33.3 \%$ for CDP/TP or E. The recurrences were null in the case of DP and enucleation of additional tumors in the pancreatic head. Therefore, the risk of recurrence increased from 0 to $11 \%$ in patients who had DP and E in comparison with patients who had DP alone. To further understand the role of each surgical procedure, we subsequently focused on patients who benefit from DP, only, or E of the body or tail of the pancreas. Our results confirm that E was associated with a higher recurrence of hypoglycemia than DP $(P=0.033)$.

However, surgical complications were found more frequently after DP than after E procedures. Even shortterm complications had occurred in $30.4 \%$ of DP procedures in contrast to $22.2 \%$ of E procedures. Finally, long-term complications, including mainly endocrine and exocrine insufficiency, had occurred after DP in $43.5 \%$ patients including new diabetes in $90 \%$ of them, compared with none of the patients who had undergone $\mathrm{E}$. This important rate of complications may be explained by the precise on-site review of all records by a single physician and a long-term follow-up.

As insulinomas often occur at a younger age than the other NENs in MEN1 patients, we think that this parameter should be cautiously evaluated within the therapeutic decision-making process and MEN1-patients informed of such complications. In addition, long-term complications will have significant morbidity and could increase with time especially in the setting of multiple MEN1-related surgical procedures. Thus, enucleation appears as an alternative in patients in whom the first goal is to achieve a low rate of comorbidity and control of hypoglycemic symptoms. However, as cure after DP was more frequently observed than after E alone, this signifies that in MEN1 patients presenting with hypoglycemic symptoms the concept of a single insulinoma should be revised and $\mathrm{E}$, if chosen, should be considered and explained to the patient as a palliative procedure even in the setting of symptomatic cure.

Until now, very limited data existed in the literature regarding the evaluation of the long-term performance of these two major surgical approaches. Only three series including more than ten patients with insulinoma and MEN1 have been published to date $(6,16,25)$. Our studies add a large body of evidence to the first series reported by the French Endocrine Tumor Group and include a systematic review of all files on site, the reports of co morbidities, and an extended follow-up in a larger number of patients. Cougard et al. (6) analyzed 41 patients with a median follow-up of 9 years: recurrent symptomatic hypoglycemia episodes were observed in eight out of $26(30.8 \%)$ patients when DP was performed but in $25 \%$ (two out of eight) of patients when $\mathrm{E}$ alone was performed. O'Riordain et al. (16) analyzed 18 patients followed up for a median of 10.3 years. Recurrent symptomatic hypoglycemia episodes were observed in $8 \%$ (one out of 12 ) of patients when DP was performed but in 20\% (one out of five) of patients when a conservative resection (minimal distal resections or tumor enucleation alone) was performed. In that study, one patient with DP had developed diabetes mellitus, whereas one patient with E had experienced pancreatic sepsis, a fistula, pancreatitis, reoperation, and permanent diabetes. Another patient had become insulin-dependent 5 years after surgery, but the type of procedure was not mentioned. More recently Bartsch et al. (25) have analyzed 13 patients (four $\mathrm{DP} \pm \mathrm{E}$; one CDP, seven E alone, and one middle pancreatectomy) followed up for a median of 13 years. Recurrent symptomatic hypoglycemia occurred in only one patient treated by enucleation only 13 years after the surgery. Six patients experienced complications including pancreatic fistula in five patients (three with E and two with DP), a wound infection in one patient with CDP, and diabetes mellitus in one patient with DP.

This study has several limitations. First, the new gradebased classification could not be evaluated. Secondly, it is retrospective and its duration accounts for the heterogeneity of the surgical procedures, the location of insulinomas by imaging techniques, and the follow-up strategy. In addition, preoperative individual findings could not be precisely retrieved. Therefore, the decisionmaking process could not be precisely analyzed making an in-depth understanding of the choice of each individual surgical approach impossible. With regards to this comment, it is also well known that the surgical procedure in MEN1 patients is affected also by the presence of other functioning pNENs and tumor growth control. In addition, no functioning imaging was performed on a routine basis. Even if, in 2014, functioning imaging has not been assigned as a definitive role regarding the choice of the best surgical strategy in insulinoma-MEN1 patients, regionalization of insulin-secreting pNENs is improving. However, despite the lack of functioning imaging in our study, the rate of insulinomas cure was as high as $82.2 \%$ 
and reached $91.3 \%$ after DP after a median post-operative follow-up of 9.0 years. This result suggests that functioning imaging could be restricted to patients in whom enucleation of other sparing parenchyma procedure is planned as well as in patients presenting with a dominant tumor within the head of the pancreas. Finally, the relatively low number of events did not allow to extensively adjust for all potential confounders, and results regarding long-term complications are not based on a survival analysis. In addition, these results should also be interpreted within the framework of a national network.

In conclusion, in the French endocrine database of MEN1-associated insulinomas, there are fewer recurrent hypoglycemic episodes after distal pancreatectomies in comparison with enucleations in the entire population but also when focusing on surgery performed on the pancreatic tail. However, enucleation might be considered as an alternative when long-term comorbidity is a major concern.

\section{Declaration of interest}

The authors declare that there is no conflict of interest that could be perceived as prejudicing the impartiality of the research reported.

\section{Funding}

This research did not receive any specific grant from any funding agency in the public, commercial or not-for-profit sector.

\section{Acknowledgements}

The authors acknowledge Lorna Saint Ange and Antoine Bennet for editing and the following physicians who provided information about their patients for this study: Chantal Cochet, Pascal D'anella, Béatrice Hamon, Stéphanie Kury-Paulin, Marie-Laure Raffin-Sanson, Frédérique Olivier, Yves Reznik, Michel Rodier, Jean-Louis Sadoul, Philippe Thieblot, and the Groupe d'étude des tumeurs endocrines (GTE) and Association Française de Chirurgie Endocrinienne (AFCE) members.

\section{References}

1 Ito T, Sasano H, Tanaka M, Osamura RY, Sasaki I, Kimura W, Takano K, Obara T, Ishibashi M, Nakao K et al. Epidemiological study of gastroenteropancreatic neuroendocrine tumors in Japan. Journal of Gastroenterology 201045 234-243. (doi:10.1007/s00535-009-0194-8)

2 Tomassetti P, Campana D, Piscitelli L, Casadei R, Santini D, Nori F, Morselli-Labate AM, Pezzilli R \& Corinaldesi R. Endocrine pancreatic tumors: factors correlated with survival. Annals of Oncology 200516 1806-1810. (doi:10.1093/annonc/mdi358)

3 Zhao YP, Zhan HX, Zhang TP, Cong L, Dai MH, Liao Q \& Cai LX. Surgical management of patients with insulinomas: result of 292 cases in a single institution. Journal of Surgical Oncology 2011103 169-174. (doi:10.1002/jso.21773)
4 Service FJ, McMahon MM, O'Brien PC \& Ballard DJ. Functioning insulinoma - incidence, recurrence, and long-term survival of patients: a 60-year study. Mayo Clinic Proceedings 199166 711-719. (doi:10.1016/ S0025-6196(12)62083-7)

5 Lo CY, Lam KY \& Fan ST. Surgical strategy for insulinomas in multiple endocrine neoplasia type I. American Journal of Surgery $1998 \mathbf{1 7 5}$ 305-307. (doi:10.1016/S0002-9610(98)00012-9)

6 Cougard P, Goudet P, Peix JL, Henry JF, Sarfati E, Proye C \& Calender A. Insulinomas in multiple endocrine neoplasia type 1 . Report of a series of 44 cases by the multiple endocrine neoplasia study group. Annales de Chirurgie 2000125 118-123. (doi:10.1016/S0001-4001(00)00112-4)

7 Piecha G, Chudek J \& Wiecek A. Multiple endocrine neoplasia type 1. European Journal of Internal Medicine 200819 99-103. (doi:10.1016/j. ejim.2007.08.004)

8 Waldmann J, Fendrich V, Habbe N, Bartsch DK, Slater EP, Kann PH, Rothmund M \& Langer P. Screening of patients with multiple endocrine neoplasia type 1 (MEN-1): a critical analysis of its value. World Journal of Surgery 200933 1208-1218. (doi:10.1007/s00268009-9983-8)

9 Grama D, Skogseid B, Wilander E, Eriksson B, Martensson H, Cedermark B, Ahren B, Kristofferson A, Oberg K, Rastad J et al. Pancreatic tumors in multiple endocrine neoplasia type 1: clinical presentation and surgical treatment. World Journal of Surgery 199216 611-618; discussion 618-619. (doi:10.1007/BF02067335)

10 Demeure MJ, Klonoff DC, Karam JH, Duh QY \& Clark OH. Insulinomas associated with multiple endocrine neoplasia type I: the need for a different surgical approach. Surgery 1991110 998-1004; discussion 1004-1005.

11 Davi MV, Boninsegna L, Dalle Carbonare L, Toaiari M, Capelli P, Scarpa A, Francia G \& Falconi M. Presentation and outcome of pancreaticoduodenal endocrine tumors in multiple endocrine neoplasia type 1 syndrome. Neuroendocrinology 201194 58-65. (doi:10.1159/000326164)

12 Mignon M, Ruszniewski P, Podevin P, Sabbagh L, Cadiot G, Rigaud D \& Bonfils S. Current approach to the management of gastrinoma and insulinoma in adults with multiple endocrine neoplasia type I. World Journal of Surgery 199317 489-497. (doi:10.1007/BF01655108)

13 Tonelli F, Fratini G, Falchetti A, Nesi G \& Brandi ML. Surgery for gastroenteropancreatic tumours in multiple endocrine neoplasia type 1: review and personal experience. Journal of Internal Medicine 2005 257 38-49. (doi:10.1111/j.1365-2796.2004.01424.x)

14 Jensen RT, Cadiot G, Brandi ML, de Herder WW, Kaltsas G, Komminoth P, Scoazec JY, Salazar R, Sauvanet A \& Kianmanesh R. ENETS Consensus Guidelines for the management of patients with digestive neuroendocrine neoplasms: functional pancreatic endocrine tumor syndromes. Neuroendocrinology 201295 98-119. (doi:10.1159/ 000335591)

15 Thompson NW. The surgical management of hyperparathyroidism and endocrine disease of the pancreas in the multiple endocrine neoplasia type 1 patient. Journal of Internal Medicine 1995238 269-280. (doi:10.1111/j.1365-2796.1995.tb00934.x)

16 O'Riordain DS, O'Brien T, van Heerden JA, Service FJ \& Grant CS. Surgical management of insulinoma associated with multiple endocrine neoplasia type I. World Journal of Surgery 199418 488-493; discussion 493-494. (doi:10.1007/BF00353743)

17 Simon D, Starke A, Goretzki PE \& Roeher HD. Reoperative surgery for organic hyperinsulinism: indications and operative strategy. World Journal of Surgery 199822 666-671; discussion 671-672. (doi:10.1007/ s002689900450)

18 Bartsch DK, Langer P, Wild A, Schilling T, Celik I, Rothmund M \& Nies C. Pancreaticoduodenal endocrine tumors in multiple endocrine neoplasia type 1: surgery or surveillance? Surgery 2000128 958-966. (doi:10.1067/msy.2000.109727)

19 Tonelli F, Fratini G, Nesi G, Tommasi MS, Batignani G, Falchetti A \& Brandi ML. Pancreatectomy in multiple endocrine neoplasia type 
1-related gastrinomas and pancreatic endocrine neoplasias. Annals of Surgery 2006244 61-70. (doi:10.1097/01.sla.0000218073.77254.62)

20 Hellman P, Goretzki P, Simon D, Dotzenrath C \& Roher HD. Therapeutic experience of 65 cases with organic hyperinsulinism. Langenbeck's Archives of Surgery 2000385 329-336. (doi:10.1007/ s004230000148)

21 Crippa S, Zerbi A, Boninsegna L, Capitanio V, Partelli S, Balzano G, Pederzoli P, Di Carlo V \& Falconi M. Surgical management of insulinomas: short- and long-term outcomes after enucleations and pancreatic resections. Archives of Surgery 2012147 261-266. (doi:10.1001/archsurg.2011.1843)

22 Ge C, Luo X, Chen X \& Guo K. Enucleation of pancreatic cystadenomas. Journal of Gastrointestinal Surgery 201014 141-147. (doi:10.1007/s11605-009-1023-3)

23 Crippa S, Bassi C, Salvia R, Falconi M, Butturini G \& Pederzoli P. Enucleation of pancreatic neoplasms. British Journal of Surgery $2007 \mathbf{9 4}$ 1254-1259. (doi:10.1002/bjs.5833)

24 Lopez CL, Waldmann J, Fendrich V, Langer P, Kann PH \& Bartsch DK. Long-term results of surgery for pancreatic neuroendocrine neoplasms in patients with MEN1. Langenbeck's Archives of Surgery 2011396 1187-1196. (doi:10.1007/s00423-011-0828-1)

25 Bartsch DK, Albers M, Knoop R, Kann PH, Fendrich V \& Waldmann J. Enucleation and limited pancreatic resection provide long-term cure for insulinoma in multiple endocrine neoplasia type 1 . Neuroendocrinology 201398 290-298. (doi:10.1159/000357779)

26 Thakker RV, Newey PJ, Walls GV, Bilezikian J, Dralle H, Ebeling PR, Melmed S, Sakurai A, Tonelli F \& Brandi ML. Clinical practice guidelines for multiple endocrine neoplasia type 1 (MEN1). Journal of Clinical Endocrinology and Metabolism 201297 2990-3011. (doi:10.1210/ jc.2012-1230)
27 Cryer PE, Axelrod L, Grossman AB, Heller SR, Montori VM, Seaquist ER \& Service FJ. Evaluation and management of adult hypoglycemic disorders: an Endocrine Society Clinical Practice Guideline. Journal of Clinical Endocrinology and Metabolism 200994 709-728. (doi:10.1210/ jc.2008-1410)

28 Kloppel G, Perren A \& Heitz PU. The gastroenteropancreatic neuroendocrine cell system and its tumors: the WHO classification. Annals of the New York Academy of Sciences 20041014 13-27. (doi:10.1196/annals. 1294.002)

29 Sobin LH, Gospodarowicz MK \& Wittekind C. TNM classification of malignant tumours. In International Union against Cancer. 7th ed. 2009. Chichester, West Sussex, UK; Hoboken, NJ: Wiley-Blackwell, 2010.

30 Rindi G, Kloppel G, Alhman H, Caplin M, Couvelard A, de Herder WW, Erikssson B, Falchetti A, Falconi M, Komminoth P et al. TNM staging of foregut (neuro)endocrine tumors: a consensus proposal including a grading system. Virchows Archiv 2006449 395-401. (doi:10.1007/ s00428-006-0250-1)

31 Norton JA, Fang TD \& Jensen RT. Surgery for gastrinoma and insulinoma in multiple endocrine neoplasia type 1 . Journal of the National Comprehensive Cancer Network 20064 148-153.

32 Giudici F, Nesi G, Brandi ML \& Tonelli F. Surgical management of insulinomas in multiple endocrine neoplasia type 1. Pancreas 201241 547-553. (doi:10.1097/MPA.0b013e3182374e08)

33 Nikfarjam M, Warshaw AL, Axelrod L, Deshpande V, Thayer SP, Ferrone CR \& Fernandez-del Castillo C. Improved contemporary surgical management of insulinomas: a 25-year experience at the Massachusetts General Hospital. Annals of Surgery 2008247 165-172. (doi:10.1097/SLA.0b013e31815792ed)

Received 15 October 2014

Revised version received 16 December 2014

Accepted 23 December 2014 